\title{
Ultrafast demagnetization of metals: Collapsed exchange versus collective excitations
}

\author{
E. Carpene* \\ IFN-CNR, Dipartimento di Fisica, Politecnico di Milano, 20133 Milano, Italy \\ H. Hedayat, F. Boschini, and C. Dallera \\ Dipartimento di Fisica, Politecnico di Milano, 20133 Milano, Italy \\ (Received 10 October 2014; revised manuscript received 27 April 2015; published 14 May 2015)
}

\begin{abstract}
We have investigated the magneto-optical response of Fe epitaxial films by femtosecond pump-probe polarimetry in a broad probe spectral region (1.8-2.6 eV). From the extrapolated photoinduced variation of the conductivity tensor, spin and charge dynamics have been disentangled. In particular, the analysis of the off-diagonal tensor element rules out any appreciable modification of the electronic band structure upon laser excitation and suggests that ultrafast demagnetization is determined by collective excitations, i.e., spin fluctuations. Our experimental evidence provides significant insight into the microscopic mechanisms governing the complex spin dynamics of metals in the subpicosecond time scale.
\end{abstract}

DOI: 10.1103/PhysRevB.91.174414

PACS number(s): 75.70.-i, 78.20.Ls, 78.47.J-

\section{INTRODUCTION}

The ultrafast loss of spin order upon femtosecond laser irradiation is a well-established experimental fact since the milestone experiment of Beaurepaire et al. in 1996 [1]. Considerable evidence has been collected in the last two decades using a variety of time-resolved techniques including the magnetooptical Kerr effect (MOKE), x-ray magnetic circular dichroism (XMCD), magnetic second harmonic generation (MSHG), spin- and angle-resolved photoemission (PES) on simple ferromagnets ( $\mathrm{Fe}, \mathrm{Co}, \mathrm{Ni}, \mathrm{Gd}$ ) [2-11], ferromagnetic alloys and multilayers [12-17], rare earth-transition metal ferrimagnets [18-22], and half-metallic oxides [23-27]. Theoretical explanations of this phenomenon have been suggested, but the underlying mechanism is not fully understood to date. Models such as phonon-assisted Elliot-Yafet spin flip [4,9], electronmagnon scattering processes [7], superdiffusive transport [28], and, more recently, dynamic exchange splitting [29] have been proposed. Besides, according to the latest time-resolved MOKE measurements on thin nickel films, it was suggested that the origin of the ultrafast demagnetization might be purely thermal [30], and simulations based on Langevin spin dynamics allowing longitudinal fluctuations have shown that spin thermalization could be achieved within $0.1 \mathrm{ps}$ [31] past the pump pulse. All these approaches capture only partially the faceted aspects of laser-induced spin dynamics, revealing the complexity and the difficulty in describing a ferromagnetic system under strong optical excitation. Although the scattering of particles (or quasiparticles such as phonons and magnons) is the established candidate to explain ultrafast demagnetization, different models lead to incomplete results. The spin dynamics in ferromagnets can be schematically represented according to two major schemes: a Heisenberg-like description of localized magnetic moments (their directions change via collective excitations), or a Stoner-like description of itinerant electrons (the atomic magnetic moment is quenched via single-particle excitations) [32]. The former successfully captures the main thermodynamic properties of a magnetic system, and the latter

*ettore.carpene@polimi.it provides a detailed description of the fundamental scattering mechanisms, but fails on the thermodynamic features, being based on zero temperature band structure computations. A bridge between these two representations has been proposed with the development of the local-band theory (LB) of itinerant ferromagnetism [33], or within the disordered local moment (DLM) theory [34], which allows the magnetization direction at the lattice site to vary randomly. These approaches resolve the localized-itinerant complementarity assuming that time and space fluctuations are driven by the magnetization vector.

From the experimental viewpoint, one of the main methods to deduce the spin dynamics in the subpicosecond time window is time-resolved MOKE. It is based on the variation of light polarization reflected by a ferromagnetic sample as determined through the Kerr angle $\Theta=\theta+i \eta$, a complex quantity given by the so-called rotation $\theta$ (real) and ellipticity $\eta$ (imaginary). The pump-probe technique with femtosecond lasers provides temporal resolution comparable to the main (quasi)particle scattering times (i.e., tens of fs), but great care must be taken in the data analysis. It has been shown that $\theta$ and $\eta$ can differ from each other on an ultrashort time scale [35] and also that photoexcited electrons can alter the interpretation of the results [27]. Only a thorough experimental analysis can disentangle the spin dynamics from the transient electronic response, providing a genuine magnetic signal.

We have investigated the optically induced effects in $\mathrm{Fe}(001)$ epitaxial films, focusing on the picosecond time window, where electrons and spins display the largest and fastest dynamics. The temporal evolution of the conductivity tensor, deduced from our measurements, suggests that ultrafast demagnetization cannot be driven by significant variations of the electronic band structure, as recently claimed [29], but is more likely determined by spin fluctuations.

The optical response of a material to an electromagnetic wave is described by the conductivity tensor $\sigma$. For cubic ferromagnetic crystals with the magnetization oriented along the $z$ axis, the latter reads

$$
\boldsymbol{\sigma}=\boldsymbol{\sigma}^{\prime}+i \boldsymbol{\sigma}^{\prime \prime}=\left(\begin{array}{ccc}
\sigma_{x x} & \sigma_{x y} & 0 \\
-\sigma_{x y} & \sigma_{x x} & 0 \\
0 & 0 & \sigma_{z z}
\end{array}\right),
$$


where the off-diagonal elements disappear in the paramagnetic state. The absorptive part of $\sigma$ is a Hermitian tensor (real diagonal and imaginary off-diagonal), while the dispersive part is anti-Hermitian (imaginary diagonal and real offdiagonal) [36]. Even in the cubic case $\sigma_{x x}$ is not strictly identical to $\sigma_{z z}$, but in iron the difference is very small and can be neglected [37]. Therefore, only two complex quantities (i.e., four real variables) are necessary to fully characterize the conductivity tensor of iron: $\sigma_{x x}^{\prime}$ (absorption, nonmagnetic), $\sigma_{x x}^{\prime \prime}$ (dispersion, nonmagnetic), $\sigma_{x y}^{\prime}$ (dispersion, magnetic), and $\sigma_{x y}^{\prime \prime}$ (absorption, magnetic). For the sake of clarity, in the following discussion we will label the components of $\sigma$ in terms of absorption and dispersion rather than real and imaginary. Thus $\sigma_{x x}^{\prime} \rightarrow \sigma_{x x}^{a}, \sigma_{x x}^{\prime \prime} \rightarrow \sigma_{x x}^{d}, \sigma_{x y}^{\prime} \rightarrow \sigma_{x y}^{d}$, and $\sigma_{x y}^{\prime \prime} \rightarrow \sigma_{x y}^{a}$.

\section{EXPERIMENTAL DETAILS AND DATA REDUCTION}

The optical analysis has been performed measuring reflectivity and the longitudinal magneto-optical Kerr effect in $50 \mathrm{~nm}$ thick $\mathrm{Fe}(001) / \mathrm{MgO}(001)$ films with an amplified Ti:sapphire laser generating 60 fs pulses centered at $800 \mathrm{~nm}$ $(1.55 \mathrm{eV})$. Time resolution has been achieved via the pumpprobe technique with the pump (probe) beam focused to a spot size of about $200 \mu \mathrm{m}(<100 \mu \mathrm{m})$ and an average impinging fluence of about $5 \mathrm{~mJ} / \mathrm{cm}^{2}$. In order to determine all the elements of the conductivity tensor, we employed either a circularly or linearly polarized incident probe and we analyzed different polarization projections $s$-polarized photons ( $s$-pol), $p$-polarized photons ( $p$-pol), or $45^{\circ}$-polarized photons $\left(45^{\circ}\right.$-pol) after reflection by the sample (details of the experimental procedure can be found in Ref. [38]). To extend the optical information in a wide spectral range, we exploited supercontinuum generation in a sapphire crystal to produce a probe beam spanning 470-690 nm in wavelength ( $\hbar \omega \approx 1.8-2.6 \mathrm{eV}$ ). The overall time resolution is determined by the duration of pump and probe pulses and is about $130 \mathrm{fs}$. The use of films thicker than the optical penetration depth $(\approx 15 \mathrm{~nm}[39])$ avoids multiple reflections, simplifying the data analysis [40].

Figure 1(a) shows the time-resolved rotation and ellipticity ( $p$-pol projection) of the Fe film, while Fig. 1(b) reports the transient reflectivity for two polarization projections ( $s$-pol and $45^{\circ}$-pol). The data refer to a probe photon energy of $2.36 \mathrm{eV}$ (525 nm wavelength). Although subpicosecond dynamics of the two reflectivity signals and of the two Kerr components $(\theta$ and $\eta$ ) are very different from each other, they all reach stable values about 1 ps past the pump pulse, indicating that local thermal equilibrium among electrons, lattice, and spins in the irradiated spot is achieved within a picosecond (we comment on the different dynamics of $\theta$ and $\eta$ in Appendix A). In order to extract quantitative information on the electronic and spin dynamics triggered by the laser pulse, a detailed analysis has been carried out. Fresnel scattering matrix formalism provides analytical expressions of the reflectivity and Kerr angle in terms of the conductivity tensor $[41,42]$. Through a numerical procedure, the optically induced dynamics of $\sigma_{x x}$ and $\sigma_{x y}$ can be deduced from the time-resolved measurements of Figs. 1(a) and 1(b) [38]. The results are reported in Figs. 1(c) $\left(\sigma_{x x}\right)$ and $1(\mathrm{~d})\left(\sigma_{x y}\right)$. The unperturbed values (at negative delay) are in excellent agreement with existing conductivity data of

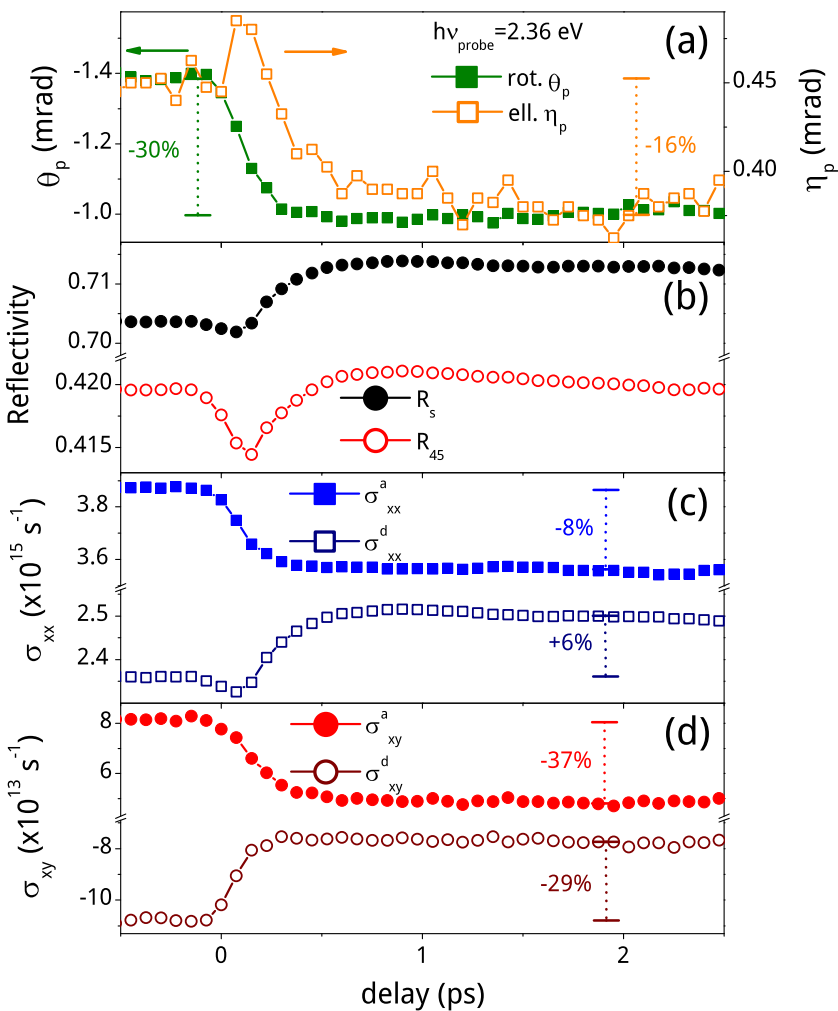

FIG. 1. (Color online) (a) Time-resolved, $p$-pol Kerr angle of the $50 \mathrm{~nm} \mathrm{Fe}(001)$ film for a probe photon energy of $2.36 \mathrm{eV}$ (solid dots: rotation; open dots: ellipticity). (b) Transient reflectivity (solid dots: $s$-pol projection; open dots: $45^{\circ}$-pol). Extrapolated dynamics of (c) the diagonal and (d) the off-diagonal elements of the conductivity tensor (solid dots: absorptive part; open dots: dispersive part).

$\mathrm{Fe}$ [37]. Laser pumping induces a reduction of both absorptive and dispersive parts of $\sigma_{x y}$ (notice that $\sigma_{x y}^{d}<0$ ), while opposite transient behaviors are observed for the two components of $\sigma_{x x}$. This implies a marginal change of the modulus $\left|\sigma_{x x}\right|$ (about $4 \%$ reduction at 2 ps delay), but a large reduction of $\left|\sigma_{x y}\right|$ (about $30 \%$ at 2 ps delay). In the following, we focus on the subpicosecond dynamics of the conductivity tensor.

\section{SUBPICOSECOND DYNAMICS OF THE CONDUCTIVITY TENSOR}

Figure 2(a) compares the prompt, pump-induced changes of $\sigma_{x x}$ and $\sigma_{x y}$. The data are normalized and rescaled to emphasize the mutual discrepancies. It is clear how each component of $\sigma$ undergoes different dynamics in the subpicosecond time window. In particular, the temporal shape of $\sigma_{x x}^{d}$ (open squares) suggests the presence of at least two opposite transient contributions to the conductivity. One of these contributions can be highlighted directly from the raw data by taking the difference between the rescaled absorptive and dispersive parts of $\sigma$ shown in Fig. 2(a): Figure 2(b) reports $\sigma_{x x}^{d}-\sigma_{x x}^{a}$ (squares) and $\sigma_{x y}^{a}-\sigma_{x y}^{d}$ (circles) (they are normalized for comparison purposes). This operation reveals the presence of a fast transient in both diagonal and off-diagonal tensor elements with an identical temporal shape and vanishing with time 




FIG. 2. (Color online) (a) Transient dynamics of the conductivity tensor [same data of Figs. 1(c) and 1(d) on a shorter time windows]: The data are normalized for comparative purposes. (b) Differences between dispersive and absorptive parts of the normalized data shown in (a) (also the differences are normalized). The dashed line is a phenomenological fit. Dynamics of (c) diagonal and (d) off-diagonal elements of $\boldsymbol{\sigma}$. The lines represent the fitting model.

constant $\tau_{p} \approx 260 \mathrm{fs}$, as deduced from the phenomenological fitting labeled " $f_{p}$ " (dashed line) [43].

A closer inspection of Fig. 2(a) helps elucidate the role and the interpretation of this fast transient component. Let us first focus on the diagonal element of the conductivity (square symbols): By comparing the dynamics of $\sigma_{x x}^{a}$ (solid squares) and $\sigma_{x x}^{d}$ (open squares), only the latter is noticeably affected by this transient in the subpicosecond regime, converging to $\sigma_{x x}^{a}$ at a longer delay. According to the results shown in Fig. 2(b) (i.e., $\sigma_{x x}^{d}-\sigma_{x x}^{a} \propto f_{p}$ ), we can therefore assume $\sigma_{x x}^{d} \propto \sigma_{x x}^{a}+\alpha f_{p}$ ( $\alpha>0$ is a fitting parameter and the relation holds for the normalized quantities). In other words, only the dispersive element is influenced by the fast transient. Concerning the off-diagonal element, $\sigma_{x y}^{d}$ (open circle) displays the fastest subpicosecond dynamics. We remind that the off-diagonal component of the conductivity is sensitive to the spin order (it disappears in the paramagnetic state), while the diagonal one probes mainly the electronic behavior. In the electric dipole approximation photons interact with electrons, but direct coupling between light and spins is unlikely and we do not expect off-diagonal elements to exhibit optically induced dynamics faster than diagonal ones. Thus, the short rise time of $\sigma_{x y}^{d}$, as compared to all other components of $\sigma$, suggests the fast transient revealed in Fig. 2(b) mostly affects the dispersive off-diagonal term, making it (apparently) faster. Similar to the diagonal case, we can therefore assume $\sigma_{x y}^{d} \propto \sigma_{x y}^{a}-\beta f_{p}$ ( $\beta>0$ is a fitting parameter), consistently satisfying the condition $\sigma_{x y}^{a}-\sigma_{x y}^{d} \propto f_{p}$.

According to the previous considerations, the $f_{p}$ component appears mainly as the dispersive part of $\sigma$, i.e., $\sigma_{x x}^{d}$ and $\sigma_{x y}^{d}$, and a quantitative interpretation of the conductivity tensor dynamics can now be established. Figures 2(c) and 2(d) show the elements of $\sigma$ [same data of Figs. 1(c) and 1(d) in a shorter time window] and the phenomenological model (lines). The absorptive parts ( $\sigma_{x x}^{a}$ and $\sigma_{x y}^{a}$, solid symbols), being unaffected by $f_{p}$, directly provide the characteristic photoinduced rise times $\tau_{e} \approx 100$ fs for the diagonal elements and $\tau_{m} \approx 180$ fs for the off-diagonal ones [44]. Although the
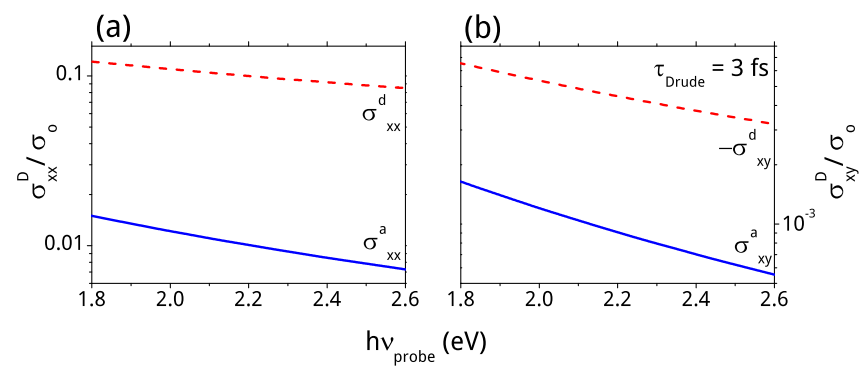

FIG. 3. (Color online) Calculated Drude-like components of (a) diagonal and (b) off-diagonal elements of the conductivity tensor of Fe assuming a Drude time $\tau=3$ fs (blue solid line: absorptive part; red dashed line: dispersive part).

value of $\tau_{e}$ is comparable to our experimental time resolution (it therefore represents an upper limit estimate), this analysis reveals different dynamics of diagonal, i.e., electronic, and off-diagonal, i.e., spin, components. The fast transient $f_{p}$ revealed in Fig. 2(b) alters only dispersive parts $\left(\sigma_{x x}^{d}\right.$ and $\sigma_{x y}^{d}$, open symbols) and it leads to a rapid and comparable reduction (about $9 \%$ ) of both diagonal and off-diagonal elements. To provide a physical interpretation of this feature, we have calculated the conductivity tensor according to the simple Drude model in the presence of the Lorentz force (in order to compute the off-diagonal part as well), here labeled $\sigma^{D}$. Although it is usually admitted that interband transitions dominate the (magneto-)optical spectra of iron [37], we tentatively attribute the $f_{p}$ component to intraband contributions, as described by the Drude model. The resulting expressions are $\sigma_{x x}^{D} \simeq \sigma_{0} /(1-i \omega \tau)$ and $\sigma_{x y}^{D} \simeq \sigma_{0} \omega_{H} \tau /(1-i \omega \tau)^{2}$, with $\omega_{H}=e H_{\mathrm{mf}} / m c$ and $\sigma_{0}=n e^{2} \tau / m$ (see Appendix $\mathrm{B}$ for details). The Drude time constant $\tau$ of iron is known from resistivity measurements and is a few femtoseconds [45], while $\hbar \omega_{H} \sim 0.1 \mathrm{eV}$ [46]. Figures 3(a) and 3(b) report the diagonal and off-diagonal Drude components, respectively, in our probe spectral range (1.8-2.6 eV) assuming an electronic scattering time $\tau=3 \mathrm{fs}$. The dispersive parts (dashed lines) are roughly an order of magnitude larger than the absorptive ones (solid lines). Besides, all elements of $\sigma^{D}$ scale proportionally to $\sigma_{0}$, i.e., to the charge density $n$. An ultrafast depletion of $n$ upon laser pumping (i.e., electrons excited from the Fermi level to higher energy) would lead to a reduction of $\sigma^{D}$ that becomes particularly evident in the dispersive element that is larger than its absorptive counterpart. The decay time $\tau_{p}$ of the fast transient reflects the charge recovery. Its extrapolated value $\tau_{p}=260 \mathrm{fs}$ is in excellent agreement with the electron-phonon coupling time in iron [7] and the $f_{p}$ component is, therefore, the signature of the electron-phonon scattering process.

The results illustrated so far refer to a selected probe energy $(2.36 \mathrm{eV})$, but the same conclusions can be drawn in the entire spectral range we investigated. In particular, the electronphonon transient affects only the dispersion, regardless of the probe wavelength. It is particularly helpful and straightforward to visualize the measured dynamics of the conductivity tensor in the complex plane (the abscissa is real, and the ordinate is imaginary). Figure 4 reports the diagonal [Fig. 4(a)] and off-diagonal [Fig. 4(b)] elements for various probe photon energies. Each curve represents the path of the component in 


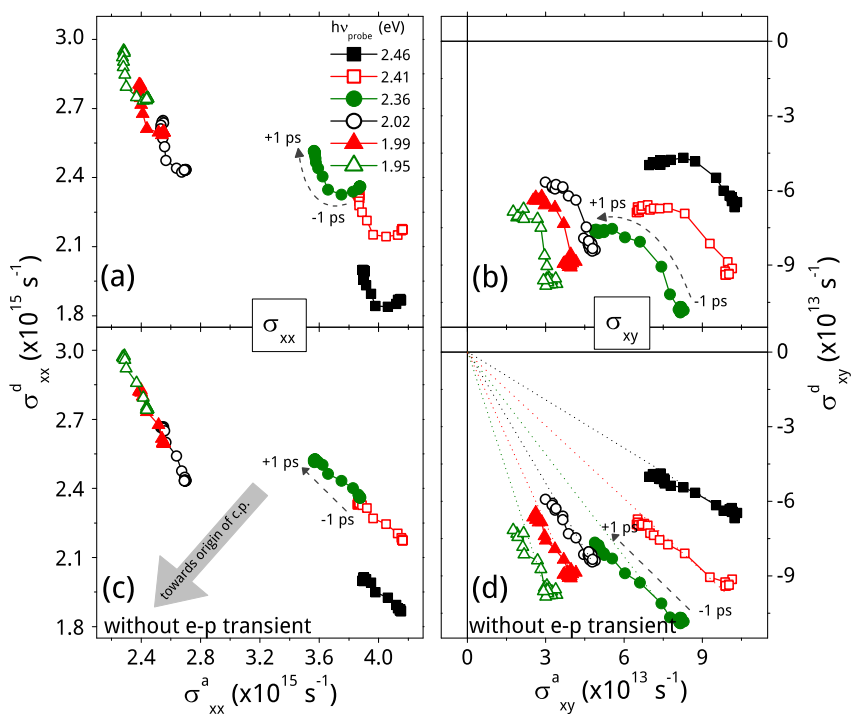

FIG. 4. (Color online) (a) Diagonal and (b) off-diagonal elements of the conductivity tensor in the complex plane (the abscissa is absorptive, and the ordinate is dispersive) for different probe energies. Each curve represents the path of the element from its unperturbed value ( -1 ps delay) to 1 ps past the pump pulse (dashed arrows indicate the time direction). (c) and (d) report the data shown in (a) and (b), respectively, after removing the transient electron-phonon components. The large arrow in (c) points towards the origin of the complex plane.

the complex plane from its unperturbed value (negative pumpprobe delay of $-1 \mathrm{ps}$ ) to 1 ps past the pump pulse, i.e., $\boldsymbol{\sigma}(t)$ for pump-probe delay $-1 \mathrm{ps} \leqslant t \leqslant+1 \mathrm{ps}$. By removing the electron-phonon contribution from $\sigma$ [i.e., by subtracting the fitting function $f_{p}$ obtained for each individual photon energy from the conductivity data, as shown in Figs. 2(c) and 2(d) for $\hbar \omega=2.36 \mathrm{eV}]$, we can evaluate its dynamics without transient electronic effects. Figures 4(c) and 4(d) show the same data of Figs. 4(a) and 4(b), respectively, after purging the electronphonon transitory part. The most interesting features emerging from Fig. 4(d) are that, regardless of the probe wavelength, the modulus of $\sigma_{x y}$ sensibly reduces $(\approx 30 \%)$ upon laser irradiation and it uniformly converges towards the origin of the complex plane. Recalling the proportionality between magnetization $M$ and the off-diagonal tensor element [a usual assumption that follows from Onsager's relations, $\sigma_{x y}(-M)=-\sigma_{x y}(M)$ [47]], the strong, photoinduced reduction of $\sigma_{x y}$ attests to the genuine ultrafast demagnetization. In contrast, the diagonal element $\sigma_{x x}$ [Fig. 4(c)] shows a marginal modulus variation: Its path in the complex plane is roughly normal to the radial direction (highlighted by the large gray arrow). Such different behaviors clearly prove that $\sigma_{x x}$ and $\sigma_{x y}$ are dominated by well-distinguished physical quantities: electrons and spins, respectively. This results qualitatively agree with previous magneto-optical measurements on $\mathrm{CoPt}_{3}$ thin films [48], in particular, the large sensitivity of the off-diagonal tensor elements to the spin order. However, the discrepancy between the pump-induced Kerr rotation and ellipticity dynamics [see Fig. 1(a)] in the subpicosecond regime seems to depend strongly on the material: It has been observed in $\mathrm{Fe}$ (present measurements) and $\mathrm{Ni}$ [35], but not in $\mathrm{CoPt}_{3}[12,48]$. Thus, relying purely on the Kerr signal to extract the spin dynamics, especially in the ultrafast regime, might be misleading and a careful analysis is required.

\section{DISCUSSION OF THE RESULTS}

With the experimental evidence in hand, we can now provide some general arguments about the demagnetization mechanism. The absorptive component of the conductivity tensor can be computed from the electronic band structure through the joint density of states (JDOS) $\mathcal{J}$. The latter is the sum of momentum matrix elements over the first Brillouin zone [49]. A simplified approach is to consider these matrix elements as constant, and the JDOS can be calculated directly from the electronic density of states (DOS) $\mathcal{D}$ as follows:

$$
\mathcal{J}_{i j}(\hbar \omega)=\int_{E_{F}-\hbar \omega}^{E_{F}} \mathcal{D}_{i}(\epsilon) \mathcal{D}_{j}(\epsilon+\hbar \omega) d \epsilon,
$$

where the indexes $i, j$ label the majority $(u)$ or minority (d) spin states, while $E_{F}$ is the Fermi level and $\hbar \omega$ is the photon energy. Spin-conserving optical transitions $(u \rightarrow u$ and $d \rightarrow d$ ) determine the absorptive part of the diagonal element $\sigma_{x x}^{a}$, which relates to the JDOS as $\mathcal{J}_{u u}(\hbar \omega)+\mathcal{J}_{d d}(\hbar \omega) \propto$ $\omega \sigma_{x x}^{a}(\hbar \omega)$. The off-diagonal element of the conductivity tensor in ferromagnets results from the interplay between the exchange interaction and spin-orbit coupling. However, and for a qualitative description, we notice that the features in the calculated spectra mimic the situation where spinflip transitions $(u \rightarrow d$ and $d \rightarrow u$ ) would take place with circular polarized light [37]. We, therefore, made the following approximation: $\omega \sigma_{x y}^{a}(\hbar \omega) \propto \mathcal{J}_{u d}(\hbar \omega)-\mathcal{J}_{d u}(\hbar \omega)$, which can be interpreted as the difference in absorption of the left and right helicity of light. Figure 5(a) shows the electronic density of states of $\mathrm{Fe}$ as calculated in Ref. [50]. Employing Eq. (2), both $\omega \sigma_{x x}^{a}(\hbar \omega)$ and $\omega \sigma_{x y}^{a}(\hbar \omega)$ [solid lines of Figs. 5(c) and 5(d), labeled $\sigma_{x x}^{a}\left(S_{0}\right)$ and $\sigma_{x y}^{a}\left(S_{0}\right)$, respectively] are estimated, obtaining an acceptable qualitative agreement with more rigorous calculations [37]. Let us now assume that ultrafast demagnetization is determined by a collapse of the exchange splitting, as recently argued in Ref. [29]: By shifting the majority and minority DOS towards the Fermi level with the only constraint that the total charge must be conserved, the overall spin polarization can be reduced. Figure 5(b) shows that a rigid shift of $+0.35 \mathrm{eV}(-0.75 \mathrm{eV})$ of the majority (minority) DOS preserves the total electron density $n_{u}+n_{d}=\int_{-\infty}^{E_{F}}\left[\mathcal{D}_{u}(\epsilon)+\mathcal{D}_{d}(\epsilon)\right] d \epsilon$, but the spin polarization $n_{u}-n_{d}=\int_{-\infty}^{E_{F}}\left[\mathcal{D}_{u}(\epsilon)-\mathcal{D}_{d}(\epsilon)\right] d \epsilon$ drops by about $30 \%$, in agreement with our measurements. The corresponding absorptive elements of the conductivity tensor are reported in Figs. 5(c) and 5(d) as dashed lines labeled $\sigma_{x x}^{a}\left(S_{1}\right)$ and $\sigma_{x y}^{a}\left(S_{1}\right)$, respectively. At this point, the demagnetization ratio $\Delta M / M$ that one should observe by means of the Kerr effect should be proportional to the ratio $\Delta \sigma_{x y}^{a} / \sigma_{x y}^{a}$. The latter can be computed from the off-diagonal conductivity terms reported in Fig. 5(d). In particular, $\Delta \sigma_{x y}^{a} / \sigma_{x y}^{a}=\left[\sigma_{x y}^{a}\left(S_{1}\right)-\sigma_{x y}^{a}\left(S_{0}\right)\right] / \sigma_{x y}^{a}\left(S_{0}\right)$. Figure 5(e) shows the calculated ratio $\Delta \sigma_{x y}^{a} / \sigma_{x y}^{a}$ versus photon energy (red solid line). The dots represent the experimental values of $\left|\Delta \sigma_{x y} / \sigma_{x y}\right|$, where the variation $\left|\Delta \sigma_{x y}\right|$ is evaluated between -1 and +1 ps delay [51]. The strong inhomogeneity 

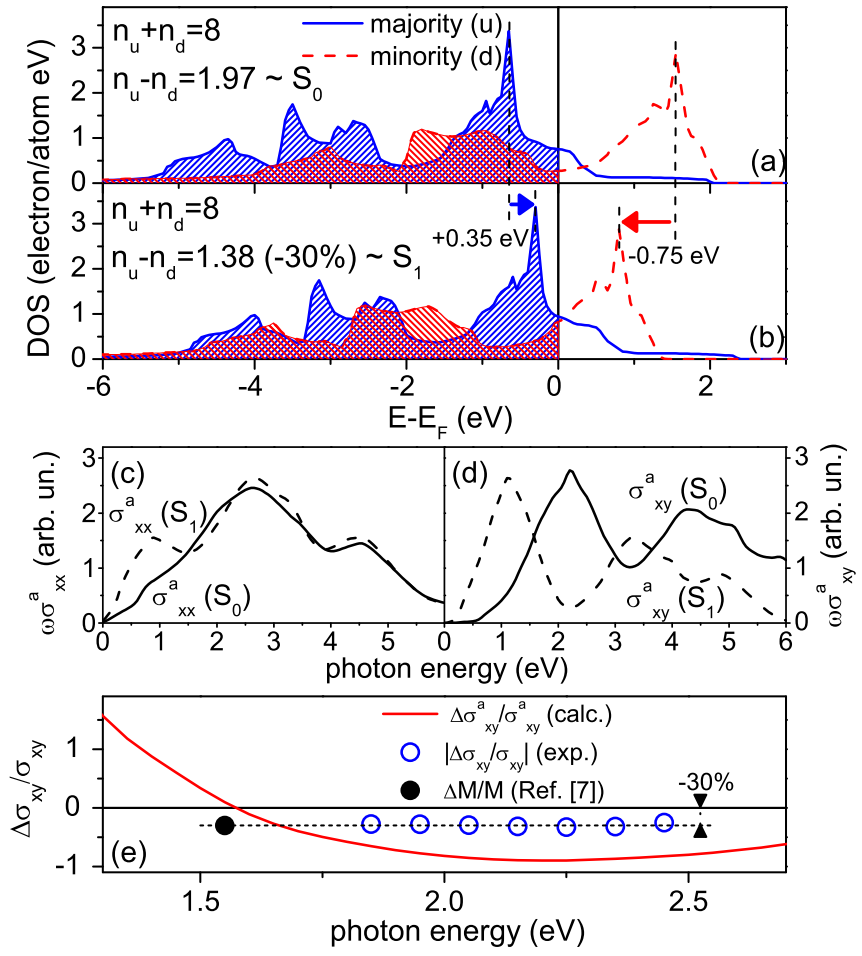

FIG. 5. (Color online) (a) Electronic density of state (DOS) of Fe, from Ref. [50] (solid line: majority spin; dashed line: minority spin). The quantities $n_{u}+n_{d}$ and $n_{u}-n_{d}$ represent the calculated total charge density and the spin polarization, respectively. (b) Density of state of Fe, assuming a collapsed exchange splitting leading to a $30 \%$ reduction of the spin polarization. Calculated absorptive part of (c) diagonal and (d) off-diagonal elements of the $\sigma$ using the DOS shown in (a) (solid lines) and in (b) (dashed lines). (e) The calculated variation $\Delta \sigma_{x y}^{a} / \sigma_{x y}^{a}$ (line) vs photon energy is compared with the experimental $\left|\Delta \sigma_{x y} / \sigma_{x y}\right|$ (dots).

of the calculated $\Delta \sigma_{x y}^{a} / \sigma_{x y}^{a}$ as a function of the photon energy clearly conflicts with our experimental evidence. Therefore, a demagnetization mechanism involving variations of the electronic structure, in particular, a collapse of the exchange, is not compatible with a wavelength-independent relative drop of $\sigma_{x y}^{a}$.

The concept of a collapsing exchange splitting (that is a direct consequence of the Stoner model of magnetism) is still rather controversial: On one side, recent femtosecond time-resolved photoemission measurements in $\mathrm{Ni}$ [3] and Gd [11] strongly support this model to explain the loss of spin order. On the other hand, a femtosecond two-photon photoemission analysis in $\mathrm{Fe}$ [52] has shown that the exchange splitting $\Delta_{\text {ex }}$ persists above the Curie point, although the long-range magnetic order breaks down at $T_{C}$. Furthermore, paramagnetic neutron scattering experiments in $\mathrm{Fe}$ have clearly shown a local magnetic moment above $T_{C}$ [53], which is not compatible with a quenched exchange interaction. This observation partially agrees with previous spin-resolved photoemission measurements versus temperature in $\mathrm{Ni}$ [54] and $\mathrm{Fe}[55,56]$ which revealed that band splitting is indeed temperature dependent, but it endures above the Curie point (in Ni) and it is observed only along some crystallographic directions (in Fe). Based on Green's function formalism,
Korenman and Prange [57] pointed out that the reduction of $\Delta_{\mathrm{ex}}$ with increasing temperature, as experimentally observed with photoemission spectroscopy, does not necessarily correspond to a collapsing exchange splitting, but is due to the appearance of satellite photoemission peaks attributed to quasiparticles (magnons). We should also mention that the exchange splitting in nickel $(\sim 0.3 \mathrm{eV}$ [54]) is comparable to the electronic thermal energy reached after a typical femtosecond pump-probe experiment $\left(k_{B} T_{e} \sim 0.1-0.2 \mathrm{eV}\right)$. This similarity in the energy scale might justify the collapse hypothesis. However, in iron $\Delta_{\mathrm{ex}} \sim 2 \mathrm{eV}$, roughly an order of magnitude larger than the electronic thermal energy, which makes the collapsing conjecture unrealistic.

Having argued that the electronic band structure should not significantly change upon laser irradiation in $\mathrm{Fe}$, an alternative path must be explored. The uniform, wavelengthindependent relative drop $\Delta \sigma_{x y} / \sigma_{x y}$ experimentally observed suggests the following explanation: Our time-resolved MOKE measurement, as much as any other experimental technique employing a light source, does not reveal the instantaneous and local value of $\sigma_{x y}$, but it rather detects the mean quantity $\left\langle\sigma_{x y}\right\rangle_{\Delta t \Delta v}$ averaged over a time interval $\Delta t$ and a volume $\Delta v$. In our case, $\Delta t$ is the experimental time resolution $(\sim 0.1 \mathrm{ps})$ and $\Delta v$ is the probed volume (proportional to the probe spot size $\Delta r \sim 0.1 \mathrm{~mm})$. In other words, the measurement integrates over space and time fluctuations of $\sigma_{x y}$. If fluctuations are faster than $\Delta t$ or take place on a distance shorter than $\Delta r$, we would inevitably observe a reduced mean value regardless of the probe wavelength. Since $\Delta r$ is roughly six orders of magnitude larger than the typical atomic distance, space fluctuations are highly justified. Beside, spin waves with a frequency larger than $1 / \Delta t$ (and thus energy $h / \Delta t>40 \mathrm{meV}$ ) are perfectly possible and their occurrence increases (exponentially) with the temperature. Collective excitations such as magnons give rise to spin fluctuations and provide a congruent explanation of all relevant experimental facts. In ferromagnetic transition metals the presence of transverse spin fluctuations (i.e., spin waves) is the dominant mechanism to explain finite temperature magnetic properties $[33,58]$. It accounts for the almost constant atomic moments at high temperature (even above $\left.T_{C}\right)$, for the low energy cost $\left(E_{\mathrm{sw}} \approx 0.05 \mathrm{eV}\right.$ is the typical energy of a spin wave [59]) required to suppress the long-range magnetic order, yet retaining the short-range order on the length scale of several lattice spacings [58], and also for the characteristic time scale of the process (as already mentioned, $t_{\mathrm{sw}} \approx h / E_{\mathrm{sw}} \approx 10^{-13} \mathrm{~s}$, completely matching the experimental evidence). Since optical excitations bring the system to a nonequilibrium electronic configuration, enhanced electron-quasiparticle scattering processes are expected. In a previous work [7] we have already suggested the possibility of a strong electron-magnon interaction as a possible path to explain ultrafast demagnetization in iron. This enhancement is triggered by the high electronic temperature reached after a femtosecond optical excitation and takes place within 100 fs. According to our present evidence, the area probed by the laser shows a reduced magnetization after an optical excitation simply because the measurement averages the enhanced spin disorder over time and space, with no need to invoke modifications of the electronic band structure. 


\section{CONCLUSIONS}

In conclusion, we have carried out a detailed time-resolved analysis of reflectivity and the magneto-optical Kerr effect in Fe films on a broad spectral range. The measurements allowed us to determine the dynamics of the conductivity tensor $\sigma$ and to disentangle charge and spin contributions. The extrapolated dynamics of $\sigma$ suggests that ultrafast demagnetization cannot be attributed to strong variations of the band structure, such as a collapsing exchange interaction. Instead, it is more likely determined by spin fluctuations, which consistently explain the observed temporal and spectral behavior of the conductivity.

\section{ACKNOWLEDGMENT}

Fondazione Cariplo (Grant 2011-0389) is gratefully acknowledged for financial support.

\section{APPENDIX A: TRANSIENT KERR ROTATION AND ELLIPTICITY}

The origin of the different rotation and ellipticity dynamics reported in Fig. 1(a) can be explained on the basis of the observed temporal behavior of the conductivity tensor. We first point out that the maximum drop of ellipticity is about $16 \%$, while the transient decrease of rotation is almost $30 \%$. The analytical expression of $\Theta$ is usually written in terms of the dielectric tensor as $\Theta=f\left(\varepsilon_{x x}\right) \times \varepsilon_{x y}$. For a probe incidence angle of $45^{\circ}$, as in our experimental setup, it can be shown that [42]

$$
f\left(\varepsilon_{x x}\right)=-\frac{\sqrt{2 \varepsilon_{x x}-1}+1}{\sqrt{2}\left(\varepsilon_{x x}-1\right)^{2} \sqrt{2 \varepsilon_{x x}-1}} .
$$

Using the relation between $\boldsymbol{\varepsilon}$ and $\boldsymbol{\sigma}$ (i.e., $\boldsymbol{\varepsilon}=\boldsymbol{I}+4 \pi i \boldsymbol{\sigma} / \omega$, with $\omega$ being the electromagnetic wave frequency), a similar expression in terms of the conductivity tensor can be obtained, i.e., $\Theta=g\left(\sigma_{x x}\right) \times \sigma_{x y}$. It is now convenient to think of these complex quantities in their polar form: $g=|g| e^{i \alpha}$ and $\sigma_{x y}=\left|\sigma_{x y}\right| e^{i \beta}$, thus $\Theta=|g|\left|\sigma_{x y}\right| e^{i \alpha+i \beta}$. If Kerr rotation and Kerr ellipticity scaled proportionally upon laser pumping, it would simply mean that the ratio $\theta / \eta$ is constant. But $\theta / \eta=\operatorname{Re}(\Theta) / \operatorname{Im}(\Theta)=\cot (\alpha+\beta)$. In other words, $\alpha+\beta$ would be constant. We have already shown (Fig. 3) that transient variations of $\sigma_{x x}$ [and predictably of $g\left(\sigma_{x x}\right)$ ] and $\sigma_{x y}$ in the complex plane are very different. Thus, to our best knowledge, there is no physical ground to expect $\alpha+\beta$ to be time invariant. On the contrary, we should expect transient rotation and ellipticity to be different, as long as charges (i.e., $\sigma_{x x}$ ) and spins (i.e., $\sigma_{x y}$ ) undergo dissimilar dynamics.

\section{APPENDIX B: DRUDE CONDUCTIVITY}

The free-electron (Drude-like) intraband contribution to the conductivity tensor in a ferromagnetic sample can be estimated from the classical Drude model in the presence of the Lorentz force,

$$
\frac{d \mathbf{p}}{d t}=-e\left(\mathbf{E}+\frac{\mathbf{p}}{m c} \times \mathbf{H}_{\mathrm{mf}}\right)-\frac{\mathbf{p}}{\tau},
$$

where $\mathbf{p},-e$, and $m$ are the electron momentum, charge, and mass, respectively, while $\tau$ is the Drude scattering time. $\mathbf{E}$ is the probe electric field and $\mathbf{H}_{\mathrm{mf}}=\lambda \mathbf{M}$ is the magnetic field in the mean-field approximation $(\lambda \approx 5000, M \approx 1700 \mathrm{G}$ in $\mathrm{Fe})$ [46]. In the following we assume $\mathbf{H}_{\mathrm{mf}}$ is oriented along the $z$ axis. By using the forms $\mathbf{p}(t)=\operatorname{Re}\left[\mathbf{p}(\omega) e^{-i \omega t}\right], \mathbf{E}(t)=$ $\operatorname{Re}\left[\mathbf{E}(\omega) e^{-i \omega t}\right]$, the previous equation becomes algebraic [45]. Recalling that the current density is defined as $\mathbf{j}=-e n \mathbf{p} / m$ ( $n$ is the carrier density), Eq. (B1) leads to the matrix form $\boldsymbol{\sigma}^{D} \cdot \mathbf{E}=\mathbf{j}$, where the nontrivial elements of the Drude-like conductivity tensor $\sigma^{D}$ are

$$
\begin{gathered}
\sigma_{x x}^{D}=\frac{\sigma_{0}(1-i \omega \tau)}{(1-i \omega \tau)^{2}+\omega_{H}^{2} \tau^{2}} \simeq \frac{\sigma_{0}}{1-i \omega \tau}=\sigma_{z z}^{D}, \\
\sigma_{x y}^{D}=\frac{\sigma_{0} \omega_{H} \tau}{(1-i \omega \tau)^{2}+\omega_{H}^{2} \tau^{2}} \simeq \frac{\sigma_{0} \omega_{H} \tau}{(1-i \omega \tau)^{2}},
\end{gathered}
$$

with $\omega_{H}=e H_{\mathrm{mf}} / m c$ and $\sigma_{0}=n e^{2} \tau / m$. Since $\hbar \omega_{H} \approx$ $0.1 \mathrm{eV} \ll \hbar \omega_{\text {probe }}(2-3 \mathrm{eV})$, the term $\omega_{H}^{2} \tau^{2}$ in $\sigma_{x x}^{D}$ and $\sigma_{x y}^{D}$ can be safely neglected.
[1] E. Beaurepaire, J.-C. Merle, A. Daunois, and J.-Y. Bigot, Phys. Rev. Lett. 76, 4250 (1996).

[2] J. Hohlfeld, E. Matthias, R. Knorren, and K. H. Bennemann, Phys. Rev. Lett. 78, 4861 (1997).

[3] H.-S. Rhie, H. A. Dürr, and W. Eberhardt, Phys. Rev. Lett. 90, 247201 (2003).

[4] B. Koopmans, J. J. M. Ruigrok, F. Dalla Longa, and W. J. M. de Jonge, Phys. Rev. Lett. 95, 267207 (2005).

[5] M. Cinchetti, M. Sánchez Albaneda, D. Hoffmann, T. Roth, J.-P. Wüstenberg, M. Krauß, O. Andreyev, H. C. Schneider, M. Bauer, and M. Aeschlimann, Phys. Rev. Lett. 97, 177201 (2006).

[6] C. Stamm, T. Kachel, N. Pontius, R. Mitzner, T. Quast, K. Holldack, S. Khan, C. Lupulescu, E. F. Aziz, M. Wietstruk, H. A. Dürr, and W. Eberhardt, Nat. Mater. 6, 740 (2007).

[7] E. Carpene, E. Mancini, C. Dallera, M. Brenna, E. Puppin, and S. De Silvestri, Phys. Rev. B 78, 174422 (2008).

[8] C. La-O-Vorakiat, M. Siemens, M. M. Murnane, H. C. Kapteyn, S. Mathias, M. Aeschlimann, P. Grychtol, R. Adam, C. M.
Schneider, J. M. Shaw, H. Nembach, and T. J. Silva, Phys. Rev. Lett. 103, 257402 (2009).

[9] B. Koopmans, G. Malinowski, F. Dalla Longa, D. Steiauf, M. Fähnle, T. Roth, M. Cinchetti, and M. Aeschlimann, Nat. Mater. 9, 259 (2010).

[10] M. Wietstruk, A. Melnikov, C. Stamm, T. Kachel, N. Pontius, M. Sultan, C. Gahl, M. Weinelt, H. A. Dürr, and U. Bovensiepen, Phys. Rev. Lett. 106, 127401 (2011).

[11] R. Carley, K. Döbrich, B. Frietsch, C. Gahl, M. Teichmann, O. Schwarzkopf, P. Wernet, and M. Weinelt, Phys. Rev. Lett. 109, 057401 (2012).

[12] J.-Y. Bigot, L. Guidoni, E. Beaurepaire, and P. N. Saeta, Phys. Rev. Lett. 93, 077401 (2004).

[13] C. Boeglin, E. Beaurepaire, V. Halté, V. López-Flores, C. Stamm, N. Pontius, H. A. Dürr, and J.-Y. Bigot, Nature (London) 465, 458 (2010).

[14] E. Turgut, C. La-o-vorakiat, J. M. Shaw, P. Grychtol, H. T. Nembach, D. Rudolf, R. Adam, M. Aeschlimann, C. M. 
Schneider, T. J. Silva, M. M. Murnane, H. C. Kapteyn, and S. Mathias, Phys. Rev. Lett. 110, 197201 (2013).

[15] J. Mendil, P. Nieves, O. Chubykalo-Fesenko, J. Walowski, T. Santos, S. Pisana, and M. Münzenberg, Sci. Rep. 4, 3980 (2014).

[16] C.-H. Lambert, S. Mangin, B. S. D. Ch.S. Varaprasad, Y. K. Takahashi, M. Hehn, M. Cinchetti, G. Malinowski, K. Hono, Y. Fainman, M. Aeschlimann, and E. E. Fullerton, Science 345, 1337 (2014).

[17] N. Moisan, G. Malinowski, J. Mauchain, M. Hehn, B. Vodungbo, J. Lüning, S. Mangin, E. E. Fullerton, and A. Thiaville, Sci. Rep. 4, 4658 (2014).

[18] C. D. Stanciu, F. Hansteen, A. V. Kimel, A. Kirilyuk, A. Tsukamoto, A. Itoh, and T. Rasing, Phys. Rev. Lett. 99, 047601 (2007).

[19] I. Radu, K. Vahaplar, C. Stamm, T. Kachel, N. Pontius, H. A. Dürr, T. A. Ostler, J. Barker, R. F. L. Evans, R. W. Chantrell, A. Tsukamoto, A. Itoh, A. Kirilyuk, T. Rasing, and A. V. Kimel, Nature (London) 472, 205 (2011).

[20] A. Kirilyuk, A. V. Kimel, and T. Rasing, Rep. Prog. Phys. 76, 026501 (2013).

[21] S. Mangin, M. Gottwald, C. H. Lambert, D. Steil, V. Uhlir, L. Pang, M. Hehn, S. Alebrand, M. Cinchetti, G. Malinowski, Y. Fainman, M. Aeschlimann, and E. E. Fullerton, Nat. Mater. 13, 286 (2014).

[22] N. Bergeard, V. López-Flores, V. Halté, M. Hehn, C. Stamm, N. Pontius, E. Beaurepaire, and C. Boeglin, Nat. Commun. 5, 3466 (2014).

[23] R. D. Averitt, A. I. Lobad, C. Kwon, S. A. Trugman, V. K. Thorsmølle, and A. J. Taylor, Phys. Rev. Lett. 87, 017401 (2001).

[24] T. Ogasawara, K. Ohgushi, Y. Tomioka, K. S. Takahashi, H. Okamoto, M. Kawasaki, and Y. Tokura, Phys. Rev. Lett. 94, 087202 (2005).

[25] Q. Zhang, A. V. Nurmikko, G. X. Miao, G. Xiao, and A. Gupta, Phys. Rev. B 74, 064414 (2006).

[26] G. M. Müller, J. Walowski, M. Djordjevic, G.-X. Miao, A. Gupta, A. V. Ramos, K. Gehrke, V. Moshnyaga, K. Samwer, J. Schmalhorst, A. Thomas, A. Hütten, G. Reiss, J. S. Moodera, and M. Münzenberg, Nat. Mater. 8, 56 (2009).

[27] E. Carpene, F. Boschini, H. Hedayat, C. Piovera, C. Dallera, E. Puppin, M. Mansurova, M. Münzenberg, X. Zhang, and A. Gupta, Phys. Rev. B 87, 174437 (2013).

[28] M. Battiato, K. Carva, and P. M. Oppeneer, Phys. Rev. Lett. 105, 027203 (2010).

[29] B. Y. Mueller, A. Baral, S. Vollmar, M. Cinchetti, M. Aeschlimann, H. C. Schneider, and B. Rethfeld, Phys. Rev. Lett. 111, 167204 (2013).

[30] T. Roth, A. J. Schellekens, S. Alebrand, O. Schmitt, D. Steil, B. Koopmans, M. Cinchetti, and M. Aeschlimann, Phys. Rev. X 2, 021006 (2012).

[31] P.-W. Ma, S. L. Dudarev, and C. H. Woo, Phys. Rev. B 85, 184301 (2012); P.-W. Ma and S. L. Dudarev, ibid. 86, 054416 (2012).

[32] A. J. Schellekens and B. Koopmans, Phys. Rev. Lett. 110, 217204 (2013).

[33] V. Korenman, J. L. Murray, and R. E. Prange, Phys. Rev. B 16, 4032 (1977); 16, 4048 (1977); 16, 4058 (1977); R. E. Prange and V. Korenman, ibid. 19, 4691 (1979); 19, 4698 (1979).
[34] A. J. Pindor, J. Staunton, G. M. Stocks, and H. Winter, J. Phys. F 13, 979 (1983); T. Ogichi, K. Terakura, and N. Hamada, ibid. 13, 145 (1983); H. Hasegawa, ibid. 13, 1915 (1983); J. Staunton, B. L. Gyorffy, A. J. Pindor, G. M. Stocks, and H. Winter, ibid. 15, 1387 (1985).

[35] B. Koopmans, M. van Kampen, J. T. Kohlhepp, and W. J. M. de Jonge, Phys. Rev. Lett. 85, 844 (2000).

[36] P. S. Pershan, J. Appl. Phys. 38, 1482 (1967).

[37] A. Delin, O. Eriksson, B. Johansson, S. Auluck, and J. M. Wills, Phys. Rev. B 60, 14105 (1999).

[38] F. Boschini, H. Hedayat, C. Piovera, C. Dallera, A. Gupta, and E. Carpene, Rev. Sci. Instrum. 86, 013909 (2015).

[39] "Optical Properties of Selected Elements," in CRC Handbook of Chemistry and Physics, Internet Version 2005, edited by D. R. Lide (CRC Press, Boca Raton, FL, 2005), http://www.hbcpnetbase.com.

[40] The drawback is the nonuniform pumping. However, the optical penetration depth in iron increases with the photon wavelength $\lambda$ : from 11 to $17 \mathrm{~nm}$, for wavelength from 470 to $800 \mathrm{~nm} \mathrm{[39].}$ The $800 \mathrm{~nm}$ pump intensity is reduced by a factor $1 / e$ at $17 \mathrm{~nm}$ below the surface. The reflected $470 \mathrm{~nm}$ probe beam must travel $2 \times 17 \mathrm{~nm}$ to "see" the effect of the pump at that depth, but its intensity drops to $e^{-34 / 11} \simeq 5 \%$. Therefore, most of the probe information comes from a much thinner layer, where pumping is more homogeneous.

[41] Š. Višňovský, Czech. J. Phys. B 34, 969 (1984).

[42] V. Antonov, B. Harmon, and A. Yeresko, Electronic Structure and Magneto-Optical Properties of Solids (Kluwer, New York, 2004).

[43] The curves in Fig. 2(b) are modeled with an exponential decay $\left(e^{-t / \tau_{p}}\right)$, while exponential saturations $\left(1-e^{-t / \tau_{e, m}}\right)$ are used in Figs. 2(c) and 2(d). All functions are convoluted with a Gaussian profile to account for the pulse duration.

[44] $\tau_{m}$ is roughly two times larger than the demagnetization time estimated in Ref. [7]. Previous measurements were based on the analysis of Kerr rotation, which might be affected by electronic effects. However, a factor of 2 does not compromise our former conclusions.

[45] N. W. Ashcroft and N. D. Mermin, Solid State Physics (Saunders College, Philadelphia, 1976).

[46] C. Kittel, Introduction to Solid State Physics (Wiley, New York, 1986).

[47] L. Onsager, Phys. Rev. 38, 2265 (1931).

[48] L. Guidoni, E. Beaurepaire, and J.-Y. Bigot, Phys. Rev. Lett. 89, 017401 (2002).

[49] M. P. Marder, Condensed Matter Physics (Wiley, New Jersey, 2010), Chap. 20.

[50] V. L. Moruzzi, J. F. Janak, and A. R. Williams, Calculated Electronic Properties of Metals (Pergamon, New York, 1978).

[51] The solid circle in Fig. 5(e) is the demagnetization observed in a $7 \mathrm{~nm}$ Fe film with pump and probe photon energies of $1.55 \mathrm{eV}$ (from Ref. [7]). Although it refers to different experimental conditions, the optically induced demagnetization is evident, whereas the calculation predicts a marginal magnetization change at this probe energy.

[52] M. Pickel, A. B. Schmidt, M. Weinelt, and M. Donath, Phys. Rev. Lett. 104, 237204 (2010). 
[53] P. J. Brown, J. Deportes, D. Givord, and K. R. A. Ziebeck, J. Appl. Phys. 53, 1973 (1982).

[54] D. E. Eastman, F. J. Himpsel, and J. A. Knapp, Phys. Rev. Lett. 40, 1514 (1978).

[55] E. Kisker, K. Schröder, M. Campagna, and W. Gudat, Phys. Rev. Lett. 52, 2285 (1984).
[56] E. Kisker, K. Schröder, W. Gudat, and M. Campagna, Phys. Rev. B 31, 329 (1985).

[57] V. Korenman and R. E. Prange, Phys. Rev. Lett. 44, 1291 (1980). [58] H. Capellmann, J. Magn. Magn. Mater. 28, 250 (1982).

[59] M. Yethiraj, R. A. Robinson, D. S. Sivia, J. W. Lynn, and H. A. Mook, Phys. Rev. B 43, 2565 (1991). 